Article

\title{
A Numerical Study of the Effects of Wind Direction on Turbine Wakes and Power Losses in a Large Wind Farm
}

\author{
Fernando Porté-Agel *, Yu-Ting Wu and Chang-Hung Chen \\ Wind Engineering and Renewable Energy Laboratory (WIRE), École Polytechnique Fédérale \\ de Lausanne (EPFL), EPFL-ENAC-IIE-WIRE, Lausanne CH-1015, Switzerland; \\ E-Mails: bulawu@gmail.com (Y.-T.W.); chen.chang-hung@epfl.ae (C.-H.C.) \\ * Author to whom correspondence should be addressed; E-Mail: fernando.porte-agel@epfl.ch.
}

Received: 14 August 2013; in revised form: 11 September 2013 / Accepted: 22 September 2013 / Published: 17 October 2013

\begin{abstract}
In this study, large-eddy simulations (LESs) were performed to investigate the effects of changing wind direction on the turbine wakes and associated power losses in the Horns Rev offshore wind farm. In the LES framework, the turbulent subgrid-scale stresses are parameterized using a tuning-free Lagrangian scale-dependent dynamic model, and the turbine-induced forces are computed using a dynamic actuator-disk model with rotation (ADM-R). This dynamic ADM-R couples blade-element theory with a turbine-specific relation between the blade angular velocity and the shaft torque to compute simultaneously turbine angular velocity and power output. A total of 67 simulations were performed for a wide range of wind direction angles. Results from the simulations show a strong impact of wind direction on the spatial distribution of turbine-wake characteristics, such as velocity deficit and turbulence intensity. This can be explained by the fact that changing the wind angle can be viewed as changing the wind farm layout relative to the incoming wind, while keeping the same wind turbine density. Of particular importance is the effect of wind direction on the distance available for the wakes to recover and expand before encountering other downwind turbines (in full-wake or partial-wake interactions), which affects the power losses from those turbines. As a result, even small changes in wind direction angle can have strong impacts on the total wind farm power output. For example, a change in wind direction of just $10^{\circ}$ from the worst-case full-wake condition is found to increase the total power output by as much as $43 \%$. This has important implications for the design of wind farms and the management of the temporal variability of their power output.
\end{abstract}


Keywords: actuator-disk model with rotation; atmospheric boundary layer; blade-element theory; Horns Rev wind farm; large-eddy simulation; power deficit; wind direction

\section{Introduction}

The production of electrical power from wind energy has experienced a remarkable expansion in the last two decades, reaching a total installed capacity of $283 \mathrm{GW}$ in 2012 and growing exponentially at an average annual rate of about $25 \%$ [1,2]. Considering the large investments being made in the wind energy sector, with many large wind farms being installed and others under planning, optimizing their design and operation is crucial to maximize their performance. Besides maximizing wind farm power output, it is important to understand and predict its variability in order to optimize its integration to the electrical grid. This involves predicting the inherently turbulent atmospheric boundary layer (ABL) flow, its spatial and temporal variability and its interaction with the wind farms. Particularly important is the prediction of multiple turbine wakes, which are known to lead to substantial power losses in wind farms, e.g., [3-9].

In wind farms, turbine wake effects on wind velocity deficits and the associated power deficits are modulated by several factors, such as terrain characteristics (e.g., surface roughness, heterogeneity and topography), wind farm characteristics (e.g., turbine type and farm layout) and atmospheric conditions, like wind direction, atmospheric stability and turbulence level. Indeed, changing wind direction can be regarded as changing wind farm layout relative to the incoming wind, while keeping the same turbine density.

Recent field studies [4,10] and numerical simulations [11,12] have shown that the power deficits in the well-studied Horns Rev offshore wind farm are strongly affected by wind direction for different wind sectors $\left( \pm 1^{\circ}, \pm 5^{\circ}, \pm 10^{\circ}, \pm 15^{\circ}\right)$ around the three selected wind direction angles of $\theta_{\text {wind }}=270^{\circ}$ (from the west), $222^{\circ}$ and $312^{\circ}$. For these wind directions, the wind is parallel to different "lines" of wind turbines, leading to what is commonly called full-wake conditions, with different streamwise distances between consecutive turbines (7.0-, 9.3- and 10.5-times the rotor diameter, respectively). In general, the longer that distance, the smaller the wake-induced power losses, particularly in the first rows of turbines. Barthelmie et al. [5] reported power data measured at the Horns Rev and the Nysted wind farms for seven wind directions, and showed an obvious increase in the power output from the downwind turbines when the incoming wind angle departs from the full-wake condition. Although all the above-mentioned studies provide valuable insights about the effects of wind direction on power deficits in wind farms, how these effects are related to the turbulent wake flow structure within the farms for a wide range of wind directions (including full-wake and partial-wake conditions) is not yet fully understood.

In this study, large-eddy simulations (LESs) are used to explore the effect of wind direction on the turbine wakes and power output from the Horns Rev wind farm. Particular emphasis is placed on understanding the impact of relatively small changes in wind direction on the wakes and their effect on the total wind farm power generation. The paper is organized as follows: Section 2 presents the LES framework and describes the different simulation cases. The simulation results are then presented and discussed in Section 3. Finally, a summary is given in Section 4. 


\section{Numerical Simulations}

\subsection{LES Framework}

We use a modified version of the LES code developed by Albertson et al. [13], Porté-Agel et al. [14], Porté-Agel [15], Stoll and Porté-Agel [16,17], Wu and Porté-Agel [12,18-20] and Porté-Agel et al. [21]. The code solves the filtered continuity equation and the filtered momentum conservation equations in rotation form. The turbulent subgrid-scale (SGS) momentum flux is modeled using a Lagrangian scale-dependent dynamic model [16], which accounts for the temporal and spatial variability of the model coefficient and its scale dependence, based on the resolved flow information without any ad hoc tuning. The numerical algorithm is written in Fortran 90 and parallelized using both Message-Passing Interface (MPI) and Open Multi-Processing (OpenMP). The main features of the numerical method can be summarized as follows: it uses a second-order Adams-Bashforth explicit scheme for time advancement and a hybrid pseudospectral finite-difference scheme for the spatial discretization. The spatial derivatives are computed using a high-order pseudospectral method in the horizontal directions and a second-order finite-difference approximation in the vertical direction. As a result, the lateral boundary conditions are periodic. The top boundary condition is set up as a flux-free condition. The bottom boundary condition requires the calculation of the instantaneous (filtered) surface shear stress as a function of the velocity field at the lowest vertical grid point, which is accomplished through the local application of Monin-Obukhov similarity theory [22].

In the LES framework, the turbine-induced forces and turbine-generated power are modeled using a recently developed actuator-disk model with rotation (ADM-R), which adopts blade-element theory to calculate the lift and drag forces (that produce thrust, rotor shaft torque and power) based on the local simulated flow and the blade characteristics [12]. In order to predict simultaneously the turbine angular velocity and the turbine-induced forces (and thus the power output), an iterative dynamic procedure is used to couple the ADM-R turbine model with a relationship between shaft torque and rotational speed. This relationship, which is unique for a given turbine model and independent of the inflow condition, is derived from simulations of a stand-alone wind turbine in conditions for which the thrust coefficient can be validated. More details regarding the dynamic ADM-R can be found in Wu and Porté-Agel [12].

This LES framework has been validated against wind-tunnel measurements of turbine wakes from both stand-alone turbines and wind farms [18,20]. Recently, the validation has been extended to the prediction of power output from the Horns Rev wind farm for three selected wind directions $\left(270^{\circ}\right.$, $222^{\circ}$ and $312^{\circ}$ ), corresponding to different full-wake conditions for which power output data are available [12]. A good agreement has been found between the measured power outputs and those simulated using the above-described LES framework. For more details on the results from the power validations, the reader is referred to Figures 4 and 5 in Wu and Porté-Agel [12].

\subsection{Case Description}

The Horns Rev offshore wind farm is selected here as a case study, since the LES framework has already been validated to predict its power output for some selected wind conditions. The wind farm is located in the eastern North Sea, around $15 \mathrm{~km}$ off the westernmost point of Denmark. It consists 
of 80 Vestas V-80 $2 \mathrm{MW}$ wind turbines within an area of about $20 \mathrm{~km}^{2}$. Each turbine has a hub height $H_{h u b}=70 \mathrm{~m}$ (above sea level) and a rotor diameter $d=80 \mathrm{~m}$. The wind farm layout has a rhomboid shape with a minimum spacing of seven rotor diameters between two consecutive turbines within each of its ten rows (approximately seven degrees turned from north-south) and eight columns (see Figure 1).

Figure 1. Schematic of the Horns Rev wind farm layout, consisting of 80 turbines arranged in ten rows (denoted as "R") and eight columns (denoted as "C"). Distances are normalized by the turbine rotor diameter $d=80 \mathrm{~m}$. Solid lines with arrows indicate selected wind directions involving full-wake conditions with different streamwise distances between consecutive wind turbines: (a) $\theta_{\text {wind }}=312^{\circ}, 295^{\circ}, 288^{\circ}, 284^{\circ}, 270^{\circ}$; and (b) $\theta_{\text {wind }}=270^{\circ}$, $256^{\circ}, 251^{\circ}, 242^{\circ}, 222^{\circ}$.

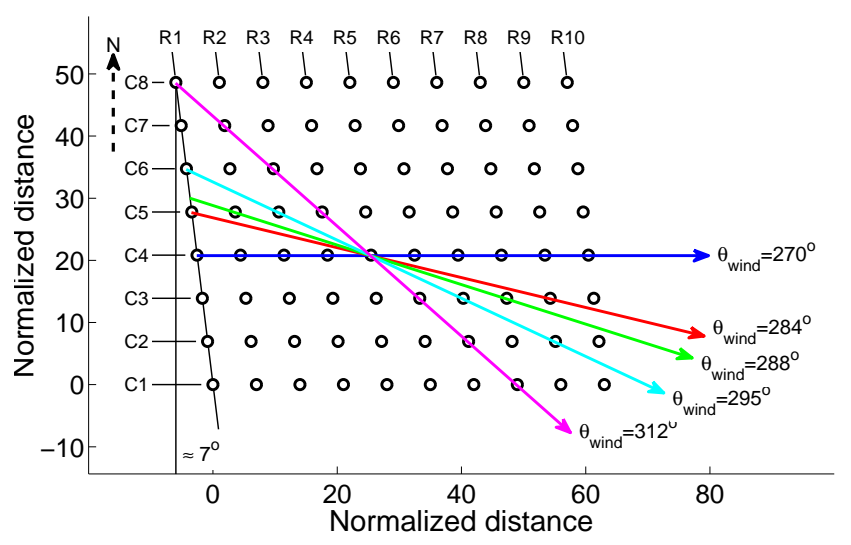

(a)

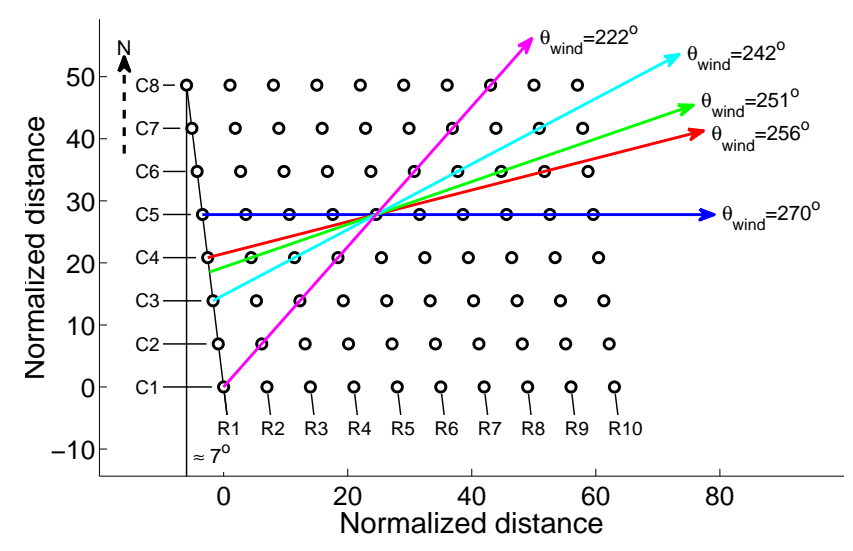

(b)

The above-described LES framework is used to perform 67 simulations of neutrally-stratified ABL flow through the Horns Rev wind farm for a wide range of wind directions (from $173^{\circ}$ to $353^{\circ}$ ). These simulations involve both full-wake and partial-wake conditions. In order to minimize flow blockage effects, the computational domain is set to a height $L_{z}=1045.9 \mathrm{~m}$ in all the simulations. A constant streamwise pressure gradient is used to drive the flow within the boundary layer, which has a height of $\delta=500 \mathrm{~m}$. This value is consistent with the boundary-layer depth observed by Peña and Gryning [23] at Horns Rev. The domain of size $\left(L_{x}, L_{y}, L_{z}\right)$ is divided uniformly into $N_{x} \times N_{y} \times N_{z}=640 \times 640 \times 128$ grid points, with a grid resolution of $\Delta_{x}, \Delta_{y}$, and $\Delta_{z}$ in the streamwise, spanwise and vertical directions, respectively. Table 1 summarizes the numerical set-up for some selected simulation cases. In this table, and throughout the paper, $S_{x}$ denotes the streamwise distance between consecutive wind turbines in the direction of the wind (under full-wake conditions), while $S_{y}$ refers to the spanwise distance between lines of wind turbines. It should be mentioned that in all the simulations, the turbine rotor diameter is covered by at least five points in the spanwise direction and nine points in the vertical direction. Based on previous resolution sensitivity studies presented in $[19,20]$, the grid resolution over the rotor considered here is well suited for our LES framework to account for the most significant characteristics of wind-turbine wakes. The dynamic ADM-R requires the specification of the turbine blade airfoil data (including chord length, twist angle, lift and drag coefficients) and the turbine torque-speed relation. Here, we use the same turbine data (see Figure 2) that were recently used to validate the LES framework through the comparison with observed turbine power data [12]. 
Table 1. Numerical set-up for neutrally-stratified atmospheric boundary layer (ABL) simulations with different incoming wind directions under full-wake conditions.

\begin{tabular}{cccccccccc}
\hline Case & $\boldsymbol{\theta}_{\boldsymbol{w i n d}}\left({ }^{\circ}\right)$ & $\boldsymbol{S}_{\boldsymbol{x}}(\mathbf{d})$ & $\boldsymbol{S}_{\boldsymbol{y}}(\mathbf{d})$ & $\boldsymbol{L}_{\boldsymbol{x}}(\mathbf{m})$ & $\boldsymbol{L}_{\boldsymbol{y}}(\mathbf{m})$ & $\boldsymbol{L}_{\boldsymbol{z}}(\mathbf{m})$ & $\boldsymbol{\Delta}_{\boldsymbol{x}}(\mathbf{m})$ & $\boldsymbol{\Delta}_{\boldsymbol{y}}(\mathbf{m})$ & $\boldsymbol{\Delta}_{\boldsymbol{z}}(\mathbf{m})$ \\
\hline HR-173 & 173 & 7.00 & 6.95 & $14,537.1$ & $9,614.5$ & $1,045.9$ & 22.71 & 15.02 & 8.24 \\
HR-222 & 222 & 9.28 & 5.24 & $13,194.9$ & $9,585.8$ & $1,045.9$ & 20.62 & 14.98 & 8.24 \\
HR-242 & 242 & 15.00 & 3.24 & $15,089.4$ & $9,302.7$ & $1,045.9$ & 23.58 & 14.54 & 8.24 \\
HR-251 & 251 & 21.30 & 2.28 & $15,400.8$ & $8,988.0$ & $1,045.9$ & 24.06 & 14.04 & 8.24 \\
HR-256 & 256 & 28.90 & 1.68 & $14,467.1$ & $9,873.4$ & $1,045.9$ & 22.60 & 14.43 & 8.24 \\
HR-270 & 270 & 7.00 & 6.95 & $14,933.3$ & $9,881.6$ & $1,045.9$ & 23.33 & 15.44 & 8.24 \\
HR-284 & 284 & 28.90 & 1.68 & $14,518.3$ & $9,323.3$ & $1,045.9$ & 22.68 & 14.57 & 8.24 \\
HR-288 & 288 & 22.90 & 2.13 & $14,850.0$ & $9,049.9$ & $1,045.9$ & 23.20 & 14.14 & 8.24 \\
HR-295 & 295 & 16.30 & 2.99 & $14,756.0$ & $9,491.9$ & $1,045.9$ & 23.06 & 14.83 & 8.24 \\
HR-312 & 312 & 10.48 & 4.64 & $16,775.2$ & $9,134.9$ & $1,045.9$ & 26.21 & 14.27 & 8.24 \\
HR-353 & 353 & 7.00 & 6.95 & $14,537.1$ & $9,361.5$ & $1,045.9$ & 22.71 & 14.63 & 8.24 \\
\hline
\end{tabular}

Figure 2. (a) Distributions of the chord length, $c(r / R)$, and twist angle, $\theta(r / R)$, along the normalized rotor radius, $r / R$; (b) measured and simulated power curves and thrust coefficient curves for the Vestas V-80 2 MW turbine; (c) relationship between the total shaft torque and the rotor angular velocity; and (d) distributions of lift and drag coefficients with respect to the angle of attack.

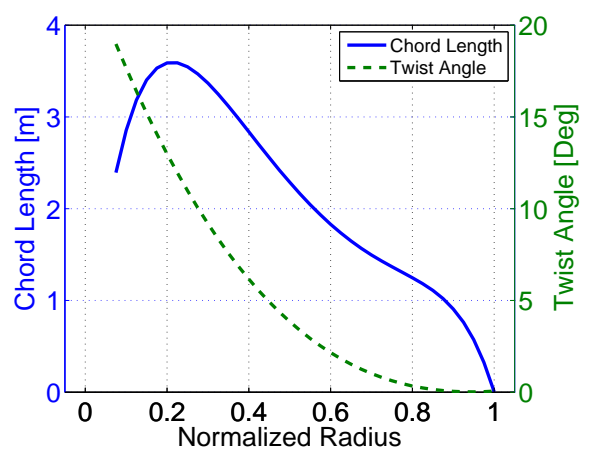

(a)

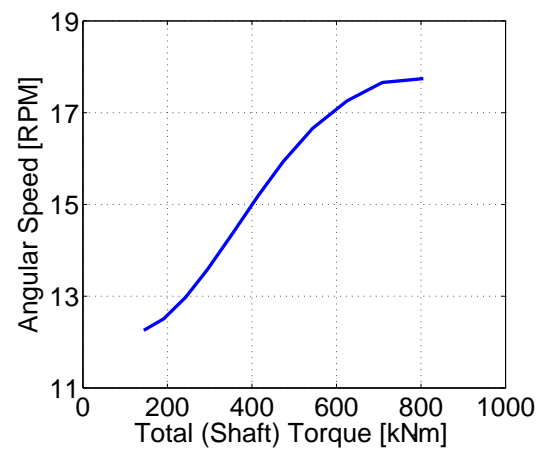

(c)

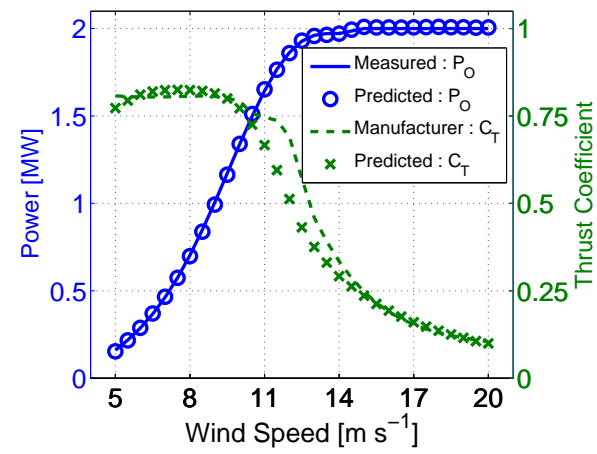

(b)

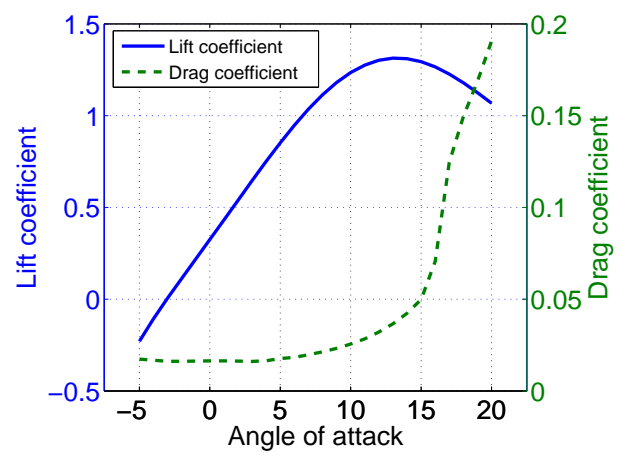

(d) 
A precursor simulation technique is adopted to generate the inflow condition for the wind farm simulations. It involves running a prior simulation of the turbulent boundary layer flow without turbines. The instantaneous velocity fields obtained from the precursor simulation are saved and later used as inflows to the wind farm simulations, using a buffer zone to adjust the flow from the very-far-wake downwind condition to that of the precursor simulation. The use of this precursor simulation technique to impose the inflow boundary condition while maintaining the accuracy of pseudospectral LES codes has been successful in previous studies of turbulent transport in urban street canopies [24], as well as flow over a steep hill [25] and flows through wind turbines [18,19] and wind farms [20]. Figure 3 shows vertical profiles of the mean velocity $(\bar{u})$, turbulence intensity and turbulent stress $\left(-\overline{u^{\prime} w^{\prime}}\right)$ obtained with the precursor simulation used in this study. The mean velocity profile is characterized by a log law with a friction velocity $u_{*}=0.442 \mathrm{~m} \mathrm{~s}^{-1}$ and an aerodynamic roughness length $z_{0}=0.05 \mathrm{~m}$. The turbulence intensity (TI) is computed as follows:

$$
T I=\frac{\sqrt{\frac{2}{3} k}}{\bar{u}_{h u b}}
$$

where $k$ is the resolved turbulence kinetic energy; $\bar{u}_{h u b}$ is the mean wind velocity at hub height; and the overbar denotes a temporal average. As shown in Figure 3, the inflow is characterized by a mean velocity at hub height of $8 \mathrm{~m} \mathrm{~s}^{-1}$ and a turbulence intensity of $7.7 \%$ at the same height. The aerodynamic roughness length used in this study was chosen to provide inflow characteristics that are in good agreement with the observed data at Horns Rev (TI $<8 \%$ at hub height when $\bar{u}_{h u b} \approx 8 \mathrm{~m} \mathrm{~s}^{-1}$ ) [4,5]. It should be noted that the LES lower boundary condition, based on Monin-Obukhov similarity, is likely to introduce some errors, as it is not able to capture all the complex interactions between the wind and the ocean wave field below. All the wind farm simulations were run for a period (physical time) of $100 \mathrm{~min}$, and the flow statistics were computed during the last $60 \mathrm{~min}$, which guarantees quasi-steady flow conditions, as well as the statistical convergence of the results presented in the next section.

Figure 3. Vertical profiles of the mean streamwise wind velocity, turbulence intensity (TI) and total (resolved plus subgrid) streamwise turbulent shear stress of the inflow used for the wind farm simulations. The inflow was obtained with a precursor simulation without turbines.
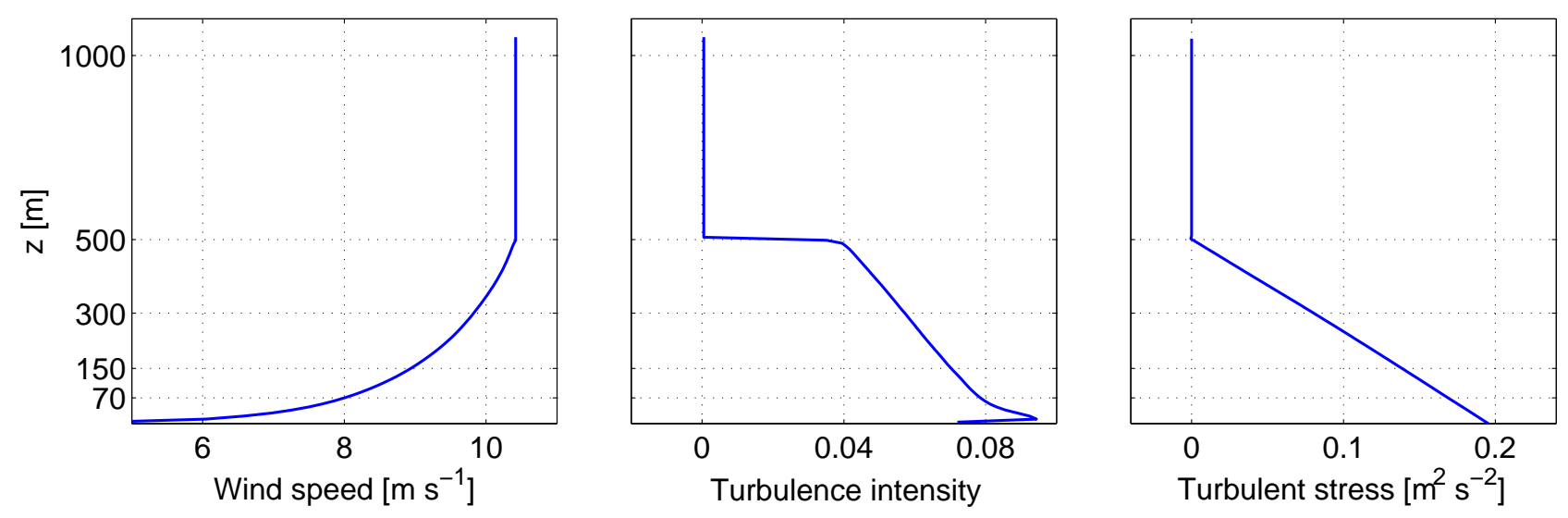


\section{Simulation Results}

The simulated total power output from the Horns Rev wind farm obtained from the 67 LESs covering a wide range of wind direction angles $\theta_{\text {wind }}$ (from $173^{\circ}$ to $353^{\circ}$ ), including full-wake and partial-wake conditions, is shown in Figure 4. Here, and throughout this paper, the simulated power is normalized by the power from an equivalent number of stand-alone wind turbines exposed to the same incoming wind condition. From Figure 4, it is clear that the minimum wind farm power occurs for the wind directions of $173^{\circ}, 270^{\circ}$ and $353^{\circ}$, which correspond to full-wake conditions with the shortest possible streamwise distance $\left(S_{x}=7.0 \mathrm{~d}\right)$ between consecutive turbines. The farm power output is slightly lower in the $270^{\circ}$ case because of the larger number of waked wind turbines in that case (72, compared with 70 for the $173^{\circ}$ and $353^{\circ}$ cases). Also evident in that figure is the presence of several local minima of power at other wind direction angles [e.g., 201 ${ }^{\circ}, 222^{\circ}, 242^{\circ}$ within the south-west (SW) $90^{\circ}$ wind sector and $295^{\circ}, 312^{\circ}, 328^{\circ}$ within the north-west (NW) $90^{\circ}$ wind sector], associated with other full-wake conditions with different streamwise distances $\left(S_{x}\right)$ between consecutive downwind turbines. Some of those wind angles are also illustrated in Figure 1. It is also interesting to note that relatively higher power outputs are obtained for partial-wake cases in the SW wind sector, compared with those in the NW wind sector. This is due to the fact that, due to its geometry, the wind farm offers a larger frontal area to the wind coming from the SW sector. This larger wind farm frontal area can accommodate a larger number of unwaked turbines, which yields higher power outputs, for specific wind direction angles.

Figure 4. Distribution of the normalized wind farm power output obtained with large-eddy simulations (LES) for a wide range of wind directions, from $173^{\circ}$ to $353^{\circ}$.

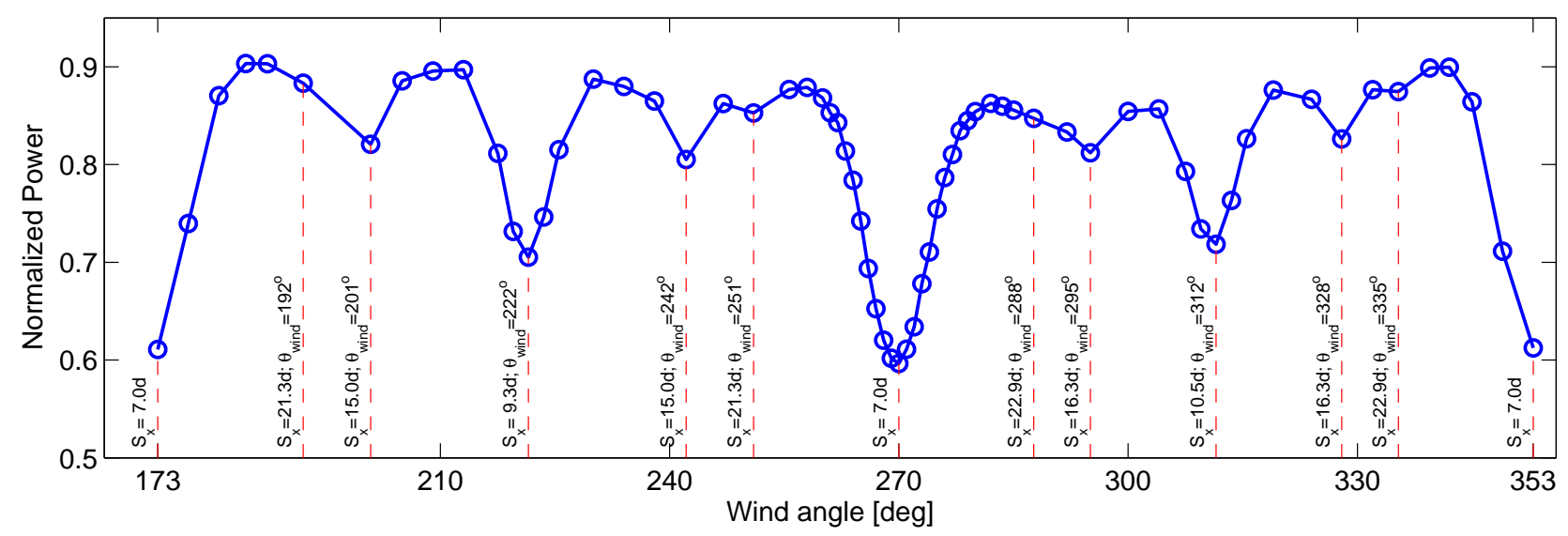

In order to better understand how the wind direction affects the structure of the multiple turbine wakes inside the farm and, in turn, the wind farm power losses depicted in Figure 4, the spatial distribution of the power outputs and the turbine wake flows are analyzed next for five selected cases. They correspond to incoming wind directions of $270^{\circ}, 284^{\circ}, 288^{\circ}, 295^{\circ}$ and $312^{\circ}$ and are illustrated in Figure 1a. The distribution of the power output is displayed in Figure 5, while the turbine wake characteristics, namely, the mean streamwise velocity, vertical velocity, streamwise turbulence intensity and horizontal momentum flux, are shown in Figures 6-9, respectively, for four of those cases. As shown in Figure 1, all these cases can be considered as full-wake conditions with different streamwise spacings $\left(S_{x}\right)$ between each wind turbine and its immediately downwind (fully-waked) neighbor. In particular, the streamwise 
turbine spacing is $7.0 d, 28.9 d, 22.9 d, 16.3 d$ and $10.5 d$ for the $270^{\circ}, 284^{\circ}, 288^{\circ}, 295^{\circ}$ and $312^{\circ}$ cases, respectively. It should be noted that, due to the fixed turbine siting density, an increase in the streamwise turbine spacing leads to a decrease in the spanwise separation between the turbine wake axes. This, in turn, increases the lateral (partial-wake) interactions and, ultimately, the power losses. This explains why, for relatively large $S_{x}$ values (larger than about $20.0 \mathrm{~d}$ ), the differences in power output between full-wake conditions and partial-wake conditions become very small, as depicted in Figure 4.

Figure 5. Spatial distribution of the normalized simulated power output for the five wind angles shown in Figure 1a: (a) power output as a function of turbine row (averaged over columns C2, C3 and C4); and (b) power output distribution along the wind direction lines (lines of turbines) shown in Figure 1a. The symbols indicate the position of the wind turbines. The streamwise distance is normalized by the turbine rotor diameter $d=80 \mathrm{~m}$.

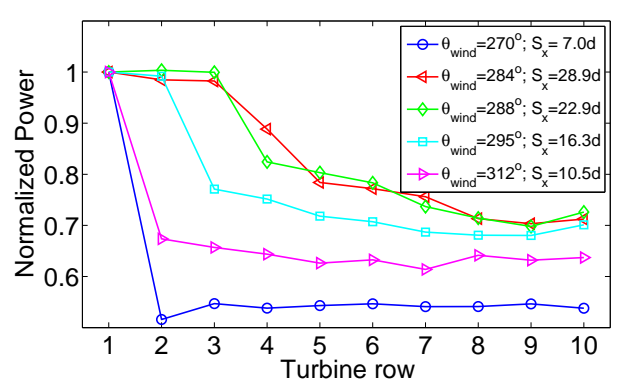

(a)

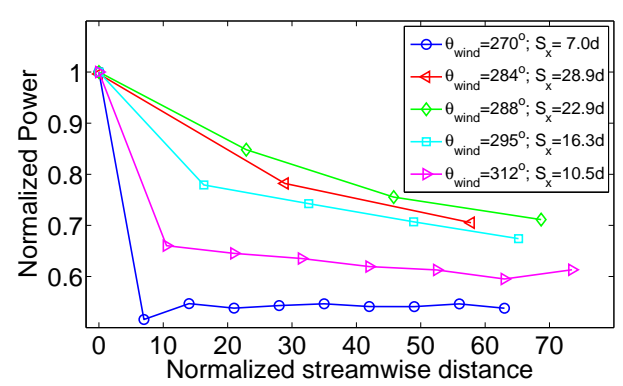

(b)

Figure 6. Contour plot of the time-averaged streamwise velocity on a horizontal plane at hub level for different incoming wind directions of (a) $270^{\circ}$; (b) $284^{\circ}$; (c) $295^{\circ}$; and (d) $312^{\circ}$. Distances are normalized by the turbine rotor diameter $d=80 \mathrm{~m}$.

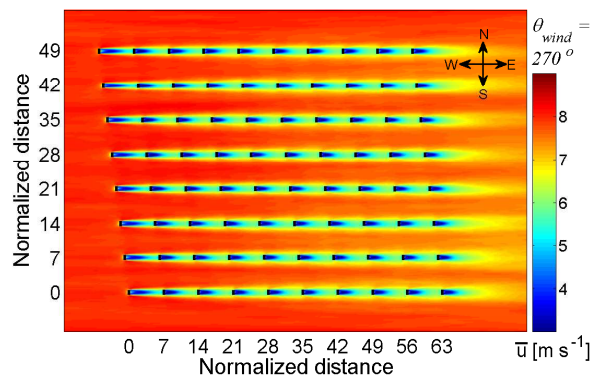

(a)

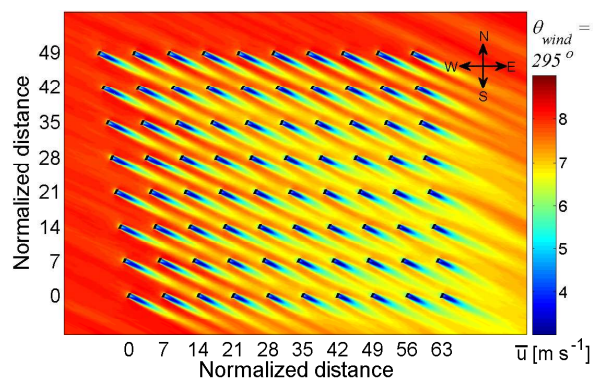

(c)

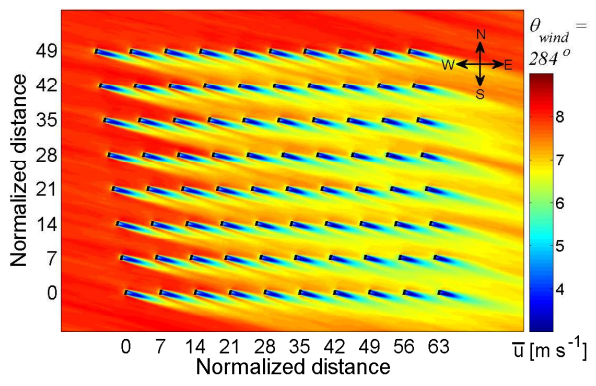

(b)

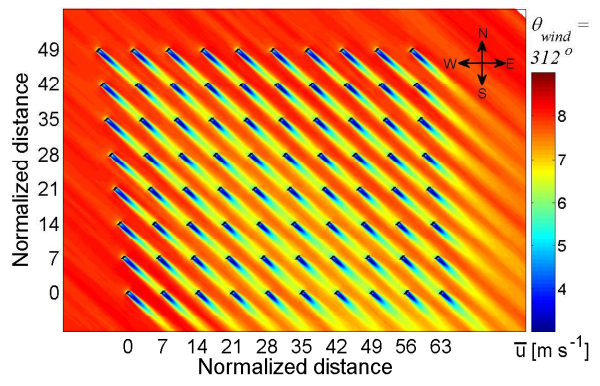

(d) 
Figure 7. Contour plot of the time-averaged vertical velocity on a horizontal plane at hub level for different incoming wind directions of (a) $270^{\circ}$; (b) $284^{\circ}$; (c) $295^{\circ}$; and (d) $312^{\circ}$.

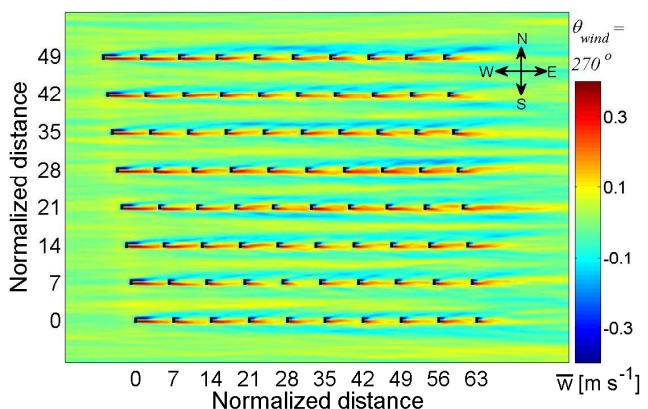

(a)

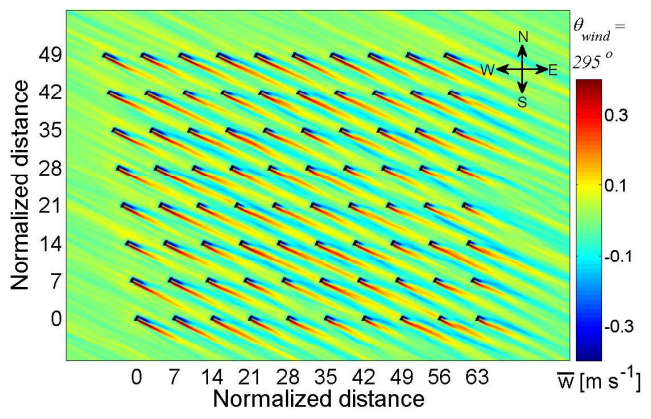

(c)

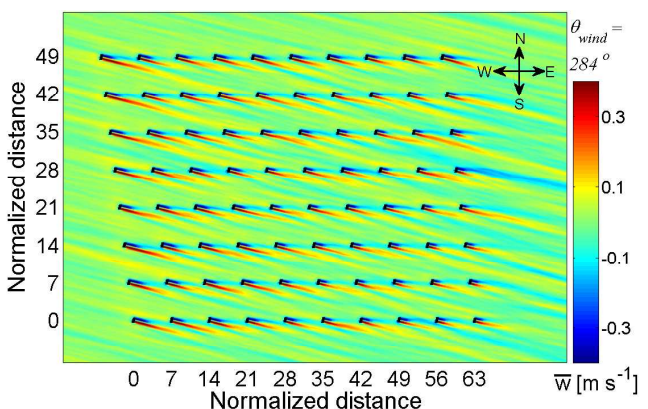

(b)

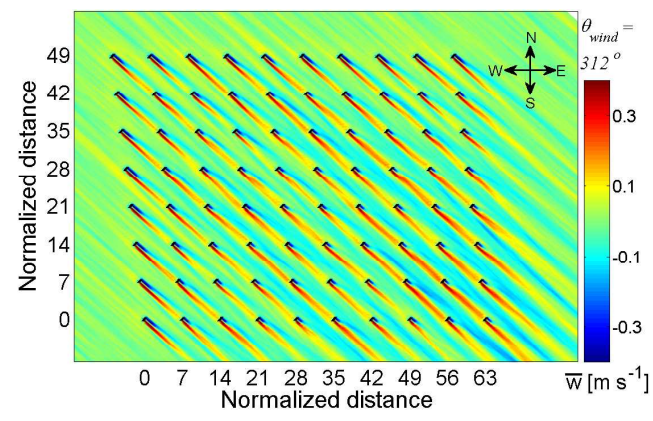

(d)

Figure 8. Contour plot of the streamwise turbulence intensity (resolved part) on a horizontal plane at hub level for different incoming wind directions of (a) $270^{\circ}$; (b) $284^{\circ}$; (c) $295^{\circ}$; and (d) $312^{\circ}$.

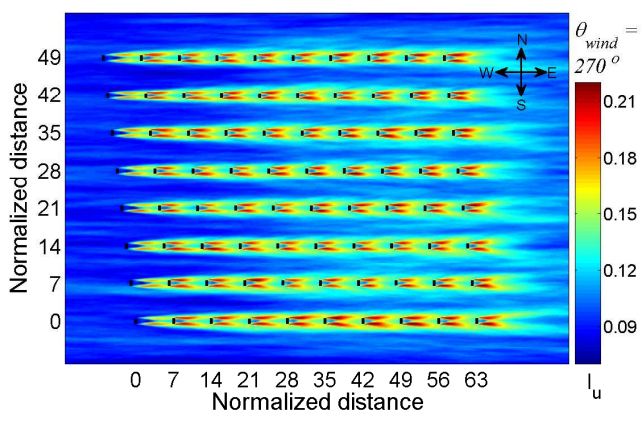

(a)

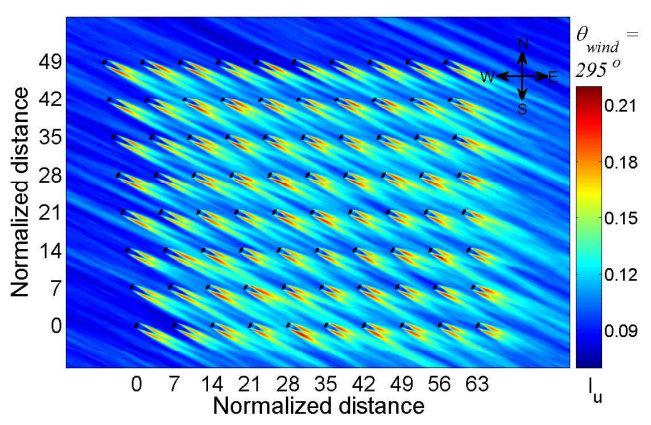

(c)

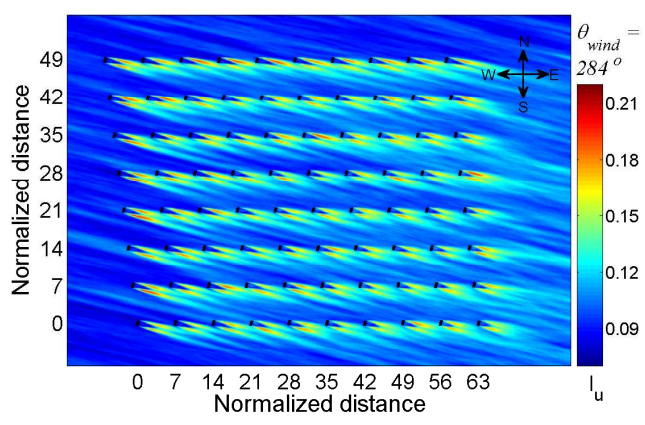

(b)

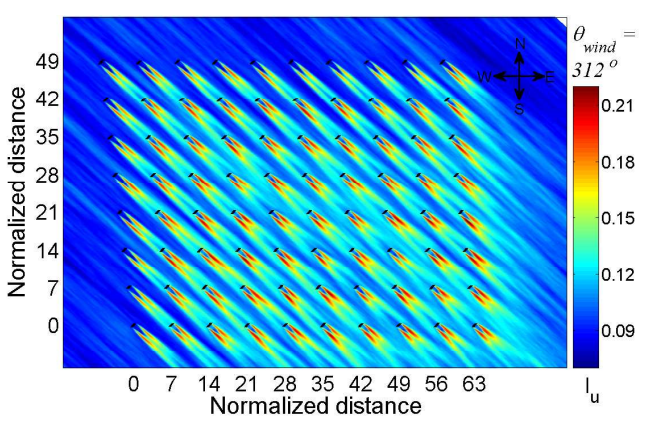

(d) 
Figure 9. Contour plot of the horizontal momentum flux [the resolved part plus the subgrid-scale (SGS) part] on a horizontal plane at hub level for different incoming wind directions of (a) $270^{\circ}$; (b) $284^{\circ}$; (c) $295^{\circ}$; and (d) $312^{\circ}$.

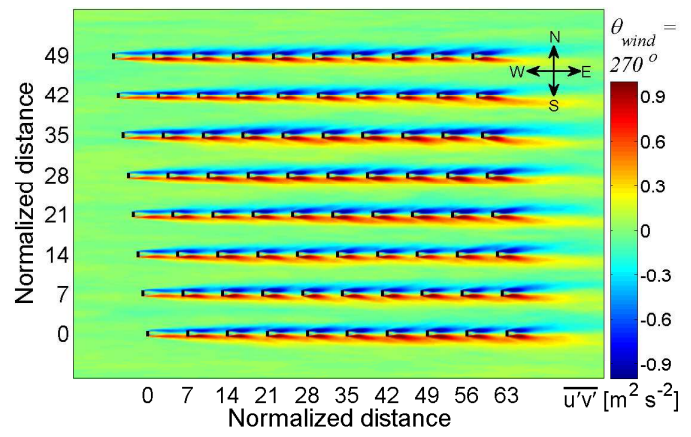

(a)

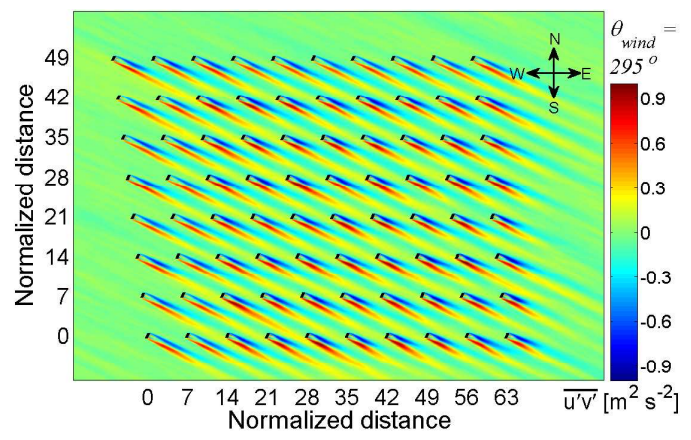

(c)

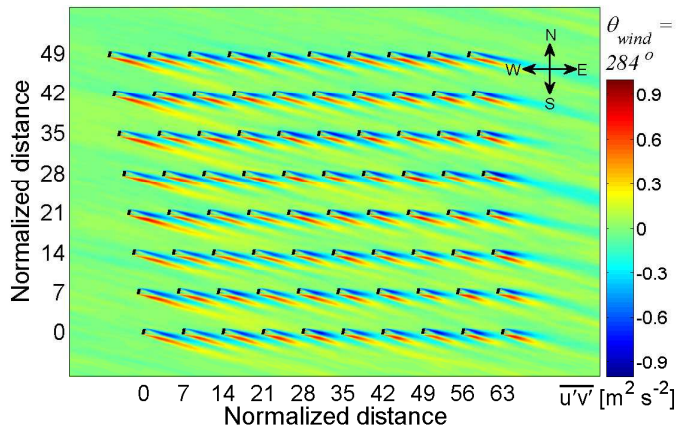

(b)

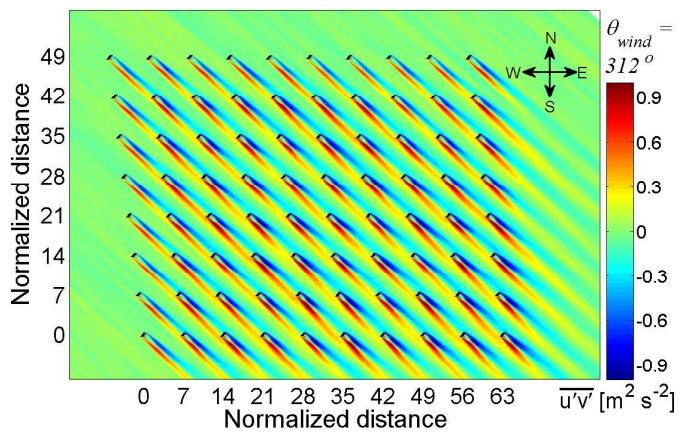

(d)

Figure 5a displays the normalized power output for the five cases under consideration as a function of the turbine row number. The results are obtained by averaging the power output from columns $\mathrm{C} 2$ to $\mathrm{C} 4$ (see Figure 1). From these results, it is evident that the spatial distribution of the turbine power output varies greatly with changing wind direction. Consistent with the results for the total farm power output shown in Figure 4, the largest power deficits throughout the farm are found for the full-wake condition that has the smallest streamwise turbine spacing $\left(S_{x}=7.0 d\right.$ for $\left.\theta_{\text {wind }}=270^{\circ}\right)$; it also corresponds to the case of the largest spanwise spacing between lines of turbines (also $S_{y}=7.0 \mathrm{~d}$ ). Here, and throughout this paper, the term 'line of turbines' is used to refer to a straight line parallel to the wind direction that connects the hubs of a series of wind turbines under full-wake conditions. It is important to note that, with a small change of wind direction of $14^{\circ}$ (from $270^{\circ}$ to $284^{\circ}$ ), the power outputs from all the turbines inside the farm increase substantially, leading to an increment of $43 \%$ in normalized wind farm power output (from 0.60 to 0.86 ), as shown in Figure 4. There are three reasons that explain this behavior:

- The relatively short streamwise distance $\left(S_{x}=7.0 d\right)$ between consecutive wind turbines in the $270^{\circ}$ case limits the wake recovery, leading to relatively large velocity deficits at the turbine locations (Figure 6a) and, in turn, large power deficits (see Figure 5) compared with the other wind direction cases.

- The relatively large spanwise distance $\left(S_{y}=7.0 d\right)$ between lines of turbines in the $270^{\circ}$ case leaves "high-speed channels" (Figure 6a), inside which a large fraction of the kinetic energy of the incoming wind is convected through the wind farm without being extracted by the turbines. 
For larger wind direction angles (particularly, 284 $)$, those channels do not exist or are much less evident, thus allowing the wind farm to extract more of the kinetic energy available in the incoming wind.

- The wind direction also determines at which row number the turbines begin to be affected by the upwind turbine wakes, which, in turn, defines the total number of waked and unwaked turbines. For example, in the $270^{\circ}$ case, a power deficit of about $50 \%$ is found already at the second turbine row and affects $90 \%$ of the wind farm, while for the $284^{\circ}$ case, the first four turbine rows, which make up about $40 \%$ of the Horns Rev wind farm, are minimally affected by turbine wakes.

Figure $5 \mathrm{~b}$ shows the power output from consecutive turbines along the wind directions following the lines shown in Figure 1. Note that the number of wind turbines in each streamwise row varies from ten to three as the streamwise spacing, $S_{x}$, increases from $7.0 d$ for $270^{\circ}$ to $28.9 d$ for $284^{\circ}$. In general, the power output from the turbines keeps decreasing along the wind direction. Unlike the $270^{\circ}$ case, for which the power output drops sharply at the second turbine row due to the short wake recovery distance (as shown in Figure 6), and remains nearly constant further downwind, the power reduction is more progressive as one moves downwind inside the wind farm for the other cases with longer streamwise distances between turbines $S_{x}$ (particularly for $S_{x}>15 d$ ). This can be explained by the fact that the smaller spanwise distance between lines of turbines, $S_{y}$, facilitates lateral wake interactions. The accumulated wakes become wider as they expand downwind (see Figure 6), leading to increasingly larger partial-wake effects and, thus, power losses when they interact laterally with the neighboring lines of turbines. In order to further illustrate the effect of wind direction on the turbine-wake structure and associated power losses, the supplementary material contains an animation showing the wind farm power output and the mean streamwise velocity at hub height for all the 67 simulated wind directions (see supplementary material).

Two-dimensional contour plots of the simulated time-averaged vertical velocity component on a horizontal plane at hub height are shown in Figure 7. The positive and negative vertical velocities obtained on both sides of the wakes highlight the ability of the LES, and in particular of the ADM-R, to reproduce the rotation of the turbine wakes. It should be noted that standard ADMs (without rotation) cannot capture wake rotation because they only account for the thrust force exerted by the turbines. As expected, the wakes rotate in the counter-clockwise direction (for an observer located upwind of the turbine), which is opposite to the standard clockwise rotation of the turbine blades. It should be noted that the vertical velocity distribution in the wakes is non-symmetric, with the positive velocity region extending further downwind compared with the negative velocity. The non-axisymmetry of the wake is a consequence of the interactions of the rotating wake flow with both the land surface and the non-uniform (logarithmic) incoming boundary layer flow. A similar non-axisymmetric distribution of the vertical velocity was reported in simulations of turbine wakes in an on-shore wind farm using the same LES framework [21].

Figure 8 shows the spatial distribution of the streamwise turbulence intensity inside the wind farm for the same four selected wind directions under consideration. The streamwise turbulence intensity is defined as the ratio between the standard deviation of the local streamwise velocity, $\sigma_{u}$, and the mean velocity of the undisturbed inflow at hub height, $\bar{u}_{h u b}$. From these results, it is clear that, as expected, turbulence intensity levels are enhanced inside the farm with respect to the incoming wind conditions 
for all cases. Different wind directions, however, lead to different levels of turbulence enhancement. In particular, the largest turbulence levels at the location of the downwind turbines are found for the $270^{\circ}$ case, and they become smaller as the streamwise distance between consecutive turbines increases. Therefore, not only the most severe full-wake cases lead to the strongest velocity deficit and power losses in the wind farm, but they also yield the highest turbulence levels at turbine level and, consequently, the largest fatigue loads on the downwind turbines.

The horizontal turbulent momentum flux, shown in Figure 9 at hub height, is negative on the right side of the wakes and positive on the left side (looking downwind) for all wind directions. This is consistent with the expected radial flux of momentum towards the wake center, which is associated with entrainment and contributes to the wake recovery. It is interesting to note the non-axisymmetric distribution of the flux, which has a relatively larger magnitude and extends further downwind on the right side, compared with the left side. The magnitude of the radial momentum flux is largest for the cases with the shortest streamwise inter-turbine distance $S_{x}$, which is consistent with the larger turbulence intensities reported in Figure 8.

It is important to point out that, as shown in Figure 10, with a change of wind direction of only $10^{\circ}$ from $270^{\circ}$, the total normalized power output from the Horns Rev wind farm increases from its minimum value of 0.60 at $270^{\circ}$ to a much higher value of about 0.86 at $270^{\circ} \pm 10^{\circ}$. Overall, these results demonstrate the significant effects that even small changes in wind direction can have on the power output from large wind farms.

Figure 10. Zoom-in of the total normalized farm power output shown in Figure 4 for a wind sector between $250^{\circ}$ and $290^{\circ}$.

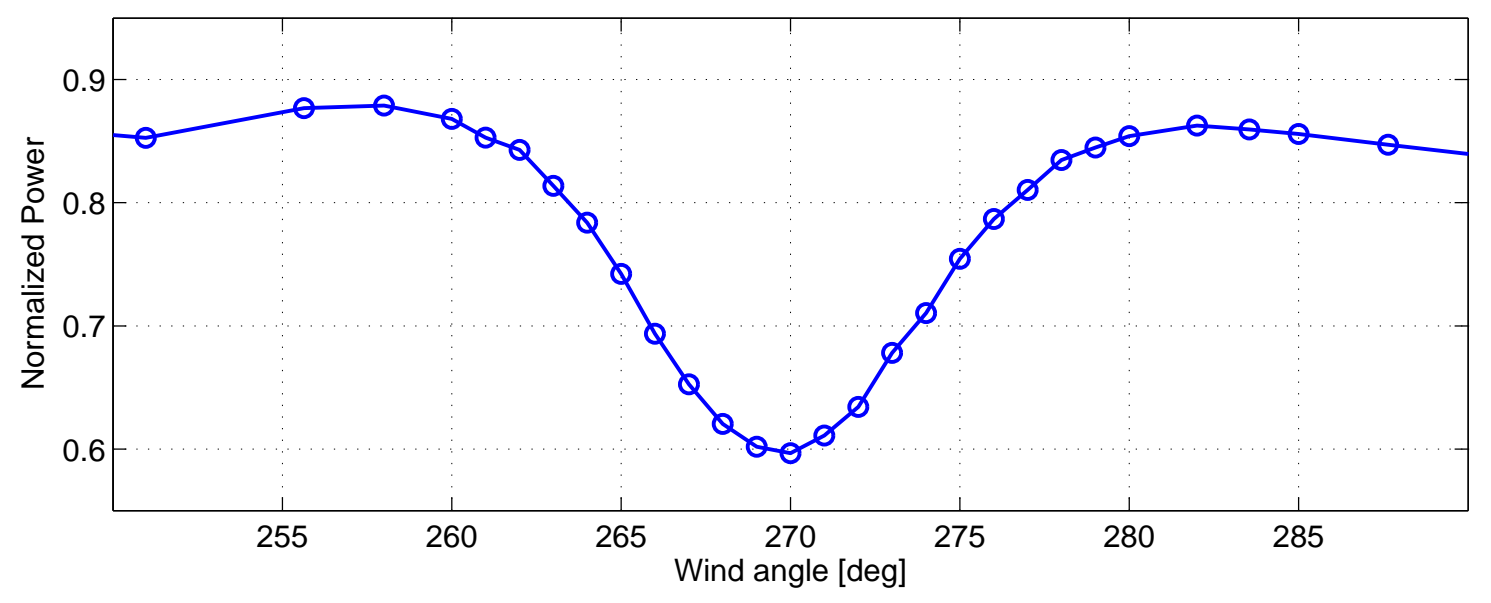

In order to better understand why a $10^{\circ}$ departure from $270^{\circ}$ yields such a large increase in power output and why it does not change much for slightly larger wind angles, we compare in Figure 11 the structure of the wakes for the $280^{\circ}$ and $284^{\circ}$ wind angles. From this figure, it is evident that, for the $280^{\circ}$ wind angle, the turbines are slightly waked by their upwind neighbors in the same row. In the $284^{\circ}$ case, the angle difference is just large enough so that the turbines are little affected by the wakes of the immediately upwind turbines. 
Figure 11. Contour plots of the time-averaged streamwise velocity $\bar{u}\left(\mathrm{~m} \mathrm{~s}^{-1}\right)$ around the third, fourth and fifth turbine columns (C3, C4 and C5 in Figure 1) at hub height for wind angles of (a) $\theta_{\text {wind }}=280^{\circ}$ and (b) $\theta_{\text {wind }}=284^{\circ}$. The black lines show the edge of the wakes (defined using the $1 \%$ velocity deficit from the undisturbed flow).

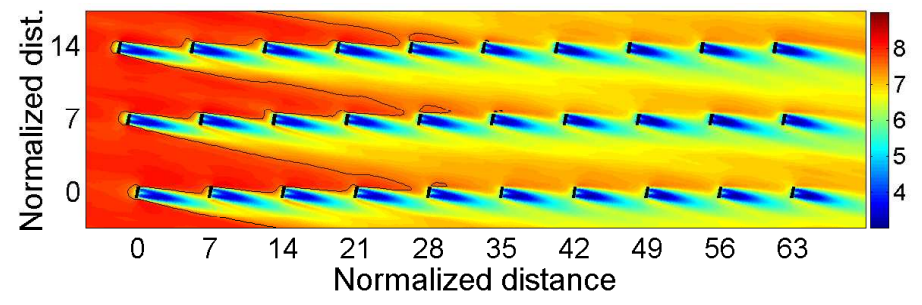

(a)

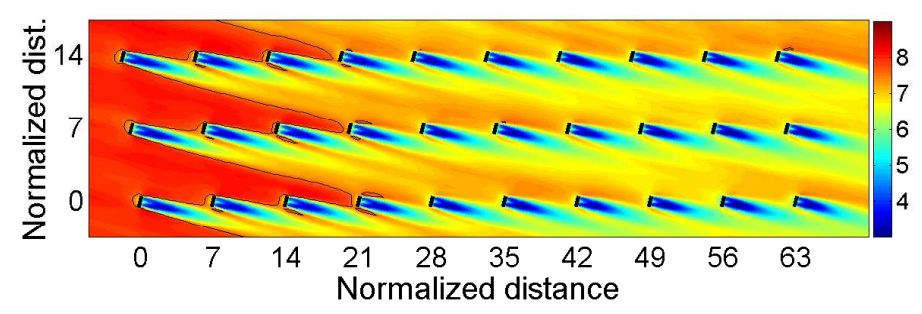

(b)

Figure 12. Schematic of the wake from a wind turbine from the first row for two selected wind direction angles: $270^{\circ}$, corresponding to the full wake condition (top); and the minimum angle $\left(270^{\circ}+\phi\right.$, where $\phi$ is $14^{\circ}$ in this case) required for the wake to have no effect on the next turbine within the same column. The blue dot-dashed line represents the wake edge, and $r_{w}$ is the radius of the wake.
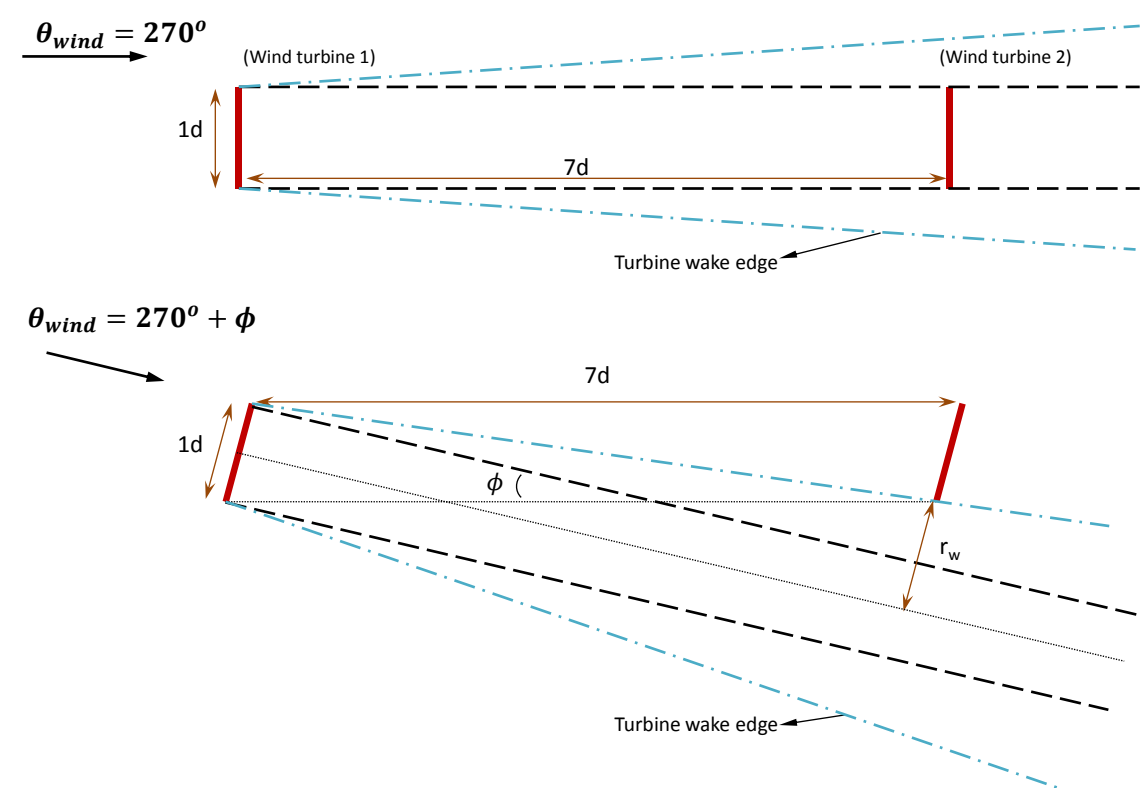

This finding is in agreement with recent results from LES [19] and also analytical modeling [26] that show that, for those inflow conditions (mean velocity and atmospheric turbulence level), the radius of 
the wake of a stand-alone V-80 turbine expands to reach $r_{w}=1.2 d$ (see Figure 12) at the position of the rotor plane of the second wind turbine at a distance of approximately $6.9 d$ downwind. In those studies, the turbine wake edge was defined based on the position at which the wake velocity deficit reaches $1 \%$ of the incoming wind velocity. That lateral position corresponds to the location of the turbine edge in the $284^{\circ}$ case. This explains why the power output from rows 2 and 3 is larger for the $284^{\circ}$ wind case compared with the $280^{\circ}$ case, for which those rows are affected by partial-wake effects (see Figure 13). Interestingly, however, this trend is reversed in rows 4-6 (see Figure 13). This is due to the fact that the larger wind angle increases the effect of the turbine wakes on the neighbor turbine columns, which are affected after the fourth row for $\theta_{\text {wind }}=284^{\circ}$, while this effect is not evident until the sixth row for the $280^{\circ}$ case (see also Figure 11). The combination of these two opposing wake effects (on the downwind turbines within the same row $v s$. on the neighboring turbine columns) makes that, for the layout and size of this wind farm, the two effects cancel out.

Figure 13. Normalized power output as a function of turbine row for wind angles of $\theta_{\text {wind }}=280^{\circ}$ and $\theta_{\text {wind }}=284^{\circ}$. The results are averaged over the seven turbine columns (C1 to $\mathrm{C} 7$ in Figure 1).

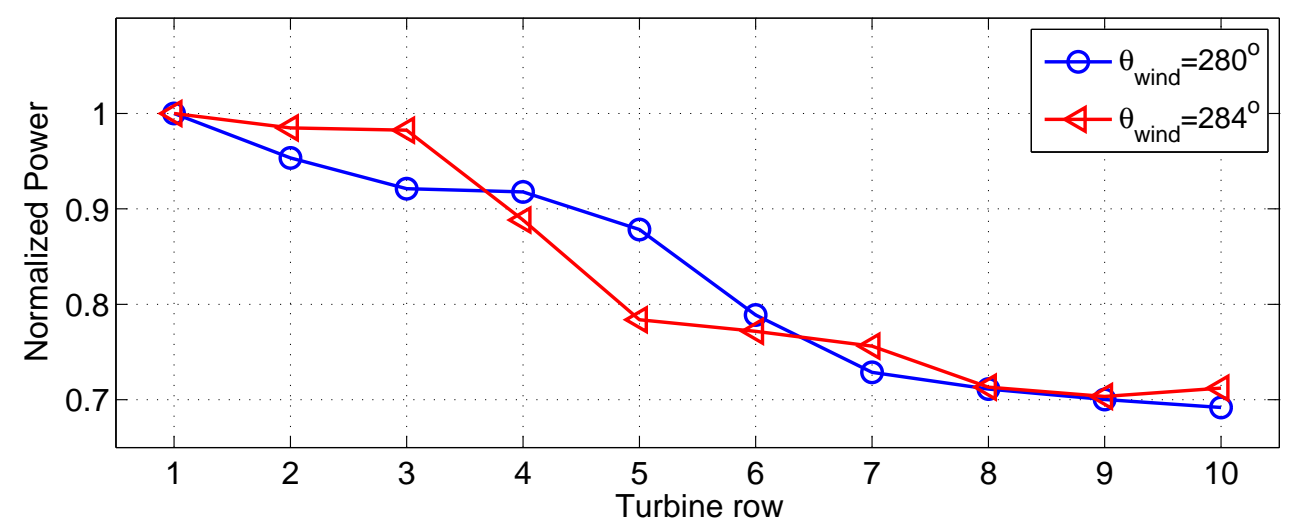

\section{Conclusions}

LESs were performed to study the effect of wind direction on the power output from a large wind farm. The Horns Rev offshore wind farm was chosen as a case study since the LES code used here was previously validated to predict power output from this farm for some selected wind conditions [12]. In this recently-developed LES framework [12,18,20,21], the SGS stresses and the turbine-induced forces are parameterized using a tuning-free Lagrangian scale-dependent dynamic model and a dynamic ADM-R, respectively. The dynamic ADM-R couples blade-element theory and a turbine-specific relation between the angular velocity and the shaft torque to compute simultaneously rotor angular velocity, shaft torque and power output.

Simulations with a series of different incoming wind directions show the strong effect of wind direction on the power output from the wind farm. To better understand that variability, it is useful to visualize the changes in wind direction as changes in wind farm layout relative to the mean wind direction, while maintaining the same turbine density (number of turbines per unit area of the farm). In particular, wind direction changes affect the relative separation between wind turbines and their 
immediately downwind (waked) neighbors, which, in turn, affects the velocity and power deficits. This explains why, for example, the smallest power output corresponds to the full-wake condition associated with the shortest streamwise distance $\left(S_{x}=7.0 \mathrm{~d}\right)$ between turbines in the direction of the wind. Moreover, in that case, part of the kinetic energy in the incoming wind passes through the wind farm through the large "channels" that separate the turbine columns, thus not being used by the wind turbines. Other angles also leading to full-wake conditions yield much smaller power losses, because the distance that the wakes have to recover before encountering the next downwind turbine is longer and the number of unwaked turbines is also larger. Furthermore, in these cases, like in many partial-wake condition cases, a reduction in the lateral distance between turbine lines $\left(S_{y}\right)$ leads to a reduction of the high-speed (untapped) channels and an increase in lateral wake interactions. These interactions, which become larger as the accumulated wakes expand downwind, lead to a more progressive increase of the power deficit inside the farm, compared with the full-wake condition cases with small $S_{x}$ (and correspondingly, large $S_{y}$ ), for which lateral wake interactions are minimal and the power deficit reaches its maximum value already at the second row of turbines.

Results from the 67 wind direction cases simulated in this study also reveal that wind farm power losses can be strongly affected by even small changes in wind direction. Particularly, in the case of the Horns Rev wind farm, the total power output is found to increase by as much as $43 \%$ when the wind direction changes by just $\pm 10^{\circ}$ from the $270^{\circ}$ angle corresponding to the worst-case (minimum power) full-wake condition. This strong variability of turbine power output, associated with small variations of wind angle, has important implications for the design of wind farms and the management of the temporal variability of wind farm power output and its integration to the electrical grid.

Future research will focus on studying the effect of additional factors, such as atmospheric stability, on wind turbine wakes and the associated power losses in wind farms. The insights provided by this and future studies on the effect of wind variability (including wind direction variability) could be used to optimize the design of wind farm layouts. In particular, they could help design alternative layouts so as to maximize power output while minimizing the variability associated with relatively small changes in wind direction.

\section{Acknowledgments}

This research was supported by the Swiss National Science Foundation (Grant 200021_132122). Computing resources were provided by the Minnesota Supercomputing Institute and the Swiss National Supercomputing Centre under project ID s306.

\section{Conflicts of Interest}

The authors declare no conflict of interest. 


\section{References}

1. Global Wind Energy Council. Global Wind Report: Annual Market Update 2012. Available online: http://www.gwec.net/wp-content/uploads/2012/06/Annual_report_2012_LowRes.pdf (accessed on 30 June 2013).

2. Mann, J.; Teilmann, J. Environmental impact of wind energy. Environ. Res. Lett. 2013, 8, doi:10.1088/1748-9326/8/3/035001.

3. Vermeer, L.J.; Sørensen, J.N.; Crespo, A. Wind turbine wake aerodynamics. Prog. Aerosp. Sci. 2003, 39, 467-510.

4. Barthelmie, R.J.; Hansen, K.; Frandsen, S.T.; Rathmann, O.; Schepers, J.G.; Schlez, W.; Phillips, J.; Rados, K.; Zervos, A.; Politis, E.S.; et al. Modelling and measuring flow and wind turbine wakes in large wind farms offshore. Wind Energy 2009, 12, 431-444.

5. Barthelmie, R.J.; Pryor, S.C.; Frandsen, S.T.; Hansen, K.S.; Schepers, J.G.; Rados, K.; Schlez, W.; Neubert, A.; Jensen, L.E.; Neckelmann, S. Quantifying the impact of wind turbine wakes on power output at offshore wind farms. J. Atmos. Ocean. Technol. 2010, 27, 1302-1317.

6. Sørensen, J.N. Aerodynamic aspects of wind energy conversion. Annu. Rev. Fluid Mech. 2011, 43, 427-448.

7. Hansen, K.S.; Barthelmie, R.J.; Jensen, L.E.; Sommer, A. The impact of turbulence intensity and atmospheric stability on power deficits due to wind turbine wakes at Horns Rev wind farm. Wind Energy 2012, 15, 183-196.

8. Gaumond, M.; Réthoré, P.E.; Ott, S.; Peña, A.; Bechmann, A.; Hansen, K.S. Evaluation of the wind direction uncertainty and its impact on wake modeling at the Horns Rev offshore wind farm. Wind Energy 2013, doi:10.1002/we.1625.

9. Hasager, C.B.; Rasmussen, L.; Peña, A.; Jensen, L.E.; Réthoré, P.E. Wind farm wake: The Horns Rev photo case. Energies 2013, 6, 696-716.

10. Barthelmie, R.J.; Rathmann, O.; Frandsen, S.T.; Hansen, K.; Politis, E.; Prospathopoulos, J.; Chaviaropoulos, P.K.; Rados, K.; Cabezón, D.; Schlez, W.; et al. Modelling and measurements of wakes in large wind farms. J. Phys. Conf. Ser. 2007, 75, doi:10.1088/1742-6596/75/1/012049.

11. Ivanell, S.; Mikkelsen, R.F.; Sørensen, J.N.; Hansen, K.S.; Henningson, D. The Impact of Wind Direction in Atmospheric BL on Interacting Wakes at Horns Rev Wind Farm. In Proceedings of the Torque 2010: The Science of Making Torque from Wind, Heraklion, Greece, 28-30 June 2010.

12. Wu, Y.T.; Porté-Agel, F. Modeling Turbine Wakes and Power Losses within a Wind Farm Using LES: An Application to the Horns Rev Offshore Wind Farm. In Proceedings of the International Conference on Aerodynamics of Offshore Wind Energy Systems and Wakes, Lyngby, Denmark, 17-19 June 2013.

13. Albertson, J.D.; Johansson, A.V.; Haritonidis, J.H.; Eckelmann, H. The fluctuating wall-shear stress and the velocity field in the viscous sublayer. Phys. Fluids 1988, 31, 1026-1033.

14. Porté-Agel, F.; Meneveau, C.; Parlange, M.B. A scale-dependent dynamic model for large-eddy simulation: Application to a neutral atmospheric boundary layer. J. Fluid Mech. 2000, 415, 261-284. 
15. Porté-Agel, F. A scale-dependent dynamic model for scalar transport in large-eddy simulations of the atmospheric boundary layer. Bound.-Layer Meteorol. 2004, 112, 81-105.

16. Stoll, R.; Porté-Agel, F. Dynamic subgrid-scale models for momentum and scalar fluxes in large-eddy simulations of neutrally stratified atmospheric boundary layers over heterogeneous terrain. Water Resour. Res. 2006, 42, doi:10.1029/2005WR003989.

17. Stoll, R.; Porté-Agel, F. Large-eddy simulation of the stable atmospheric boundary layer using dynamic models with different averaging schemes. Bound.-Layer Meteorol. 2008, 126, 1-28.

18. Wu, Y.T.; Porté-Agel, F. Large-eddy simulation of wind-turbine wakes: Evaluation of turbine parametrisations. Bound.-Layer Meteorol. 2011, 138, 345-366.

19. Wu, Y.T.; Porté-Agel, F. Atmospheric turbulence effects on wind-turbine wakes: An LES study. Energies 2012, 5, 5340-5362.

20. Wu, Y.T.; Porté-Agel, F. Simulation of turbulent flow inside and above wind farms: Model validation and layout effects. Bound.-Layer Meteorol. 2013, 146, 181-205.

21. Porté-Agel, F.; Wu, Y.T.; Lu, H.; Conzemius, R.J. Large-eddy simulation of atmospheric boundary layer flow through wind turbines and wind farms. J. Wind Eng. Ind. Aerodyn. 2011, 99, 154-168.

22. Stoll, R.; Porté-Agel, F. Effect of roughness on surface boundary conditions for large-eddy simulation. Bound.-Layer Meteorol. 2006, 118, 169-187.

23. Peña, A.; Gryning, S.E. Charnock's roughness length model and non-dimensional wind profiles over the sea. Bound.-Layer Meteorol. 2008, 128, 191-203.

24. Tseng, Y.; Meneveau, C.; Parlange, M.B. Modeling flow around bluff bodies and predicting urban dispersion using large eddy simulation. Environ. Sci. Technol. 2006, 40, 2653-2662.

25. Wan, F.; Porté-Agel, F. Large-eddy simulation of stably-stratified flow over a steep hill. Bound.-Layer Meteorol. 2011, 138, 367-384.

26. Bastankhah, M.; Porté-Agel, F. A New Analytical Model for Wind-Turbine Wakes. In Proceedings of the International Conference on Aerodynamics of Offshore Wind Energy Systems and Wakes, Lyngby, Denmark, 17-19 June 2013.

(c) 2013 by the authors; licensee MDPI, Basel, Switzerland. This article is an open access article distributed under the terms and conditions of the Creative Commons Attribution license (http://creativecommons.org/licenses/by/3.0/). 